\title{
Clinical Characteristics of Sleep-Disordered Breathing in Subacute Phase of Stroke
}

\author{
Hyunkyu Jeon, MD, Min Kyun Sohn, MD, PhD, Minsoo Jeon, MD, Sungju Jee, MD, PhD \\ Department of Rehabilitation Medicine, Chungnam National University Hospital, Daejeon, Korea
}

\begin{abstract}
Objective To assess the frequency and severity of sleep-disordered breathing (SDB) in subacute stroke patients in Korea.

Methods We consecutively enrolled subacute stroke patients who were transferred to the Department of Rehabilitation Medicine from February 2016 to August 2016. The inclusion criteria were as follows: diagnosis of the first onset of cerebral infarction or hemorrhage in the brain by computed tomography or magnetic resonance imaging; patients between 18 and 80 years old; and patients admitted within 7 days to 6 months after stroke onset. We evaluated baseline clinical data on patients' admission to the Department of Rehabilitation Medicine. We assessed demographic data, stroke severity, neurologic impairment, cognition and quality of life. We used the Epworth Sleepiness Scale to assess quality of sleep. We used a portable polysomnography to detect SDB.

Results Of the 194 stroke patients, 76 patients enrolled in this study. We evaluated and included 46 patients in the outcome analysis. The mean apnea-hypopnea index (AHI) was $24.2 \pm 17.0$ and 31 patients $(67.4 \%)$ exhibited an AHI $\geq 15$. Those in the SDB group showed a higher National Institutes of Health Stroke Scale, lower Functional Ambulation Category, lower Korean version of Modified Barthel Index, and lower EuroQol five dimensions questionnaire (EQ-5D) at admission. Prevalence and clinical characteristics of SDB did not show significant differences among stroke types or locations.

Conclusion SDB is common in subacute stroke patients. SDB must be evaluated after a stroke, particularly in patients presenting severe neurologic impairment.
\end{abstract}

Keywords Rehabilitation, Sleep, Sleep apnea syndromes, Stroke

\section{INTRODUCTION}

Sleep-disordered breathing (SDB) is characterized by recurrent episodes of nocturnal hypoxemia and causes sympathetic activation and cardiovascular distress [1]. SDB is generally associated with poor sleep quality, excessive daytime sleepiness, and decreased quality of life [2-5]. The prevalence of SDB is much higher in acute

Received October 19, 2016; Accepted January 4, 2017

Corresponding author: Sungju Jee

Department of Rehabilitation Medicine, Chungnam National University Hospital, 282 Munhwa-ro, Jung-gu, Daejeon 35015, Korea. Tel: +82-42-3382423, Fax: +82-42-338-2461, E-mail: drjeesungju@hanmail.net

ORCID: Hyunkyu Jeon (http://orcid.org/0000-0001-6186-2868); Min Kyun Sohn (http://orcid.org/0000-0002-2548-545X); Minsoo Jeon (http://orcid. org/0000-0003-2205-3210); Sungju Jee (http://orcid.org/0000-0002-9400-9609).

(c) This is an open-access article distributed under the terms of the Creative Commons Attribution Non-Commercial License (http://creativecommons.org/ licenses/by-nc/4.0) which permits unrestricted noncommercial use, distribution, and reproduction in any medium, provided the original work is properly cited. Copyright (c) 2017 by Korean Academy of Rehabilitation Medicine 
ischemic stroke patients than in the general population, which implies an association between SDB and stroke [69]. Moreover, there is evidence to suggest that the presence of SDB in stroke patients is associated with poor functional status and high mortality $[6,10,11]$.

Once a stroke has occurred, sleep apnea can aggravate functional disabilities due to nocturnal hypoxia recurrence and sleep fragmentation, as well as reduced cardiac output and cerebral perfusion [12-14]. Consequently, in stroke patients, SDB may contribute to prolonged poststroke hospitalization and rehabilitation [12]. Obstructive sleep apnea (OSA) can be effectively treated with continuous positive airway pressure, but OSA is often left undiagnosed. When left untreated, OSA can contribute to decreased recovery from stroke $[3,10]$.

The mechanisms linking SDB with stroke are complex. These mechanisms include endothelial damage, atherosclerosis, hypercoagulability, inflammatory and hemodynamic changes, paradoxical embolization and the development of cardiac arrhythmias [15]. SDB is an independent risk factor for stroke [16]. Conversely, stroke may contribute to SDB onset or aggravate premorbid SDB [17].

Previous studies have suggested that sleep apnea more likely precedes the onset of stroke. For instance, a large population-based study showed that OSA may be an independent risk factor for stroke [18]. Bassetti et al. [19] found an equal prevalence of sleep apnea in patients following transient ischemic attack (TIA) and completed stroke. In another study, Parra et al. [20] observed that the number of central apneas decreased at 3 months after stroke onset, while the number of obstructive apneas did not change. These findings suggest that acute stroke predisposes to central sleep apnea (CSA), and OSA probably precedes the onset of stroke. However, such previous data were mainly associated with acute phase ischemic stroke. Data regarding the subacute phase of stroke are lacking and evidences of hemorrhagic stroke are much more limited.

The in-laboratory polysomnography is the gold standard test for SDB [21]. However, due to constraints regarding access, cost, and patient unwillingness to sleep overnight in a laboratory setting, this device is often not feasible for many stroke and TIA patients. In this instance, a portable polysomnography is feasible for use in a post-stroke inpatient setting [22]. The present study was performed to estimate the prevalence and severity of SDB in Korean patients with subacute stroke and to investigate the association between SDB and stroke type, stroke severity, and functional outcome.

\section{MATERIALS AND METHODS}

\section{Subjects}

We consecutively enrolled all subacute stroke patients who were transferred to the Department of Rehabilitation Medicine from February 2016 to August 2016. The inclusion criteria were as follows: (1) diagnosis of the first onset of cerebral infarction or hemorrhage in the brain by computed tomography or magnetic resonance imaging; (2) patients between 18 and 80 years old; (3) patients admitted within 7 days to 6 months after stroke onset; and (4) patients providing their informed consent. We excluded patients with any of the following: (1) history of previous stroke; (2) history of traumatic brain damage or brain tumor; (3) a baseline oxygen saturation of $<95 \%$; (4) presence of acute or chronic cardiopulmonary diseases that affect pulmonary function; (5) presence of neuromuscular disease (e.g., amyotrophic lateral sclerosis and myasthenia gravis); and (6) unstable medical condition disabling completion of the clinical trial. The Institutional Review Board of Chungnam National University Hospital approved this study (No. 2015-12-012-005).

\section{Clinical evaluation}

\section{Measurements on admission}

We assessed demographic and clinical data, stroke severity, neurologic function, cognition and quality of life on admission to the Department of Rehabilitation Medicine. Basic demographic and clinical data included age, gender, body weight, height, systolic and diastolic blood pressure, and underlying diseases. All subjects were questioned regarding their cardiovascular risk factors. This information was provided by the patients or their relatives. The body mass index (BMI) was calculated for all subjects. Stroke types were classified as ischemic or hemorrhagic. The location of stroke lesions was classified as supratentorial, brainstem or cerebellum. Stroke severity was assessed by the National Institutes of Health Stroke Scale (NIHSS). Functional disability was assessed by a Modified Rankin Scale (mRS), Korean version of Modified Barthel Index (K-MBI) and the Functional Ambulation Category (FAC). Cognitive impairment was 
assessed using the Korean version of Mini-Mental State Examination (K-MMSE) and the Korean version of Montreal Cognitive Assessment (MoCA-K). Quality of life was evaluated using the EuroQol five dimensions questionnaire (EQ-5D). Dysphagia was considered as the difficulty in swallowing solid food.

\section{Measurements at discharge}

We estimated stroke outcome by the K-MBI, mRS, and NIHSS at discharge from the hospital. We obtained the length of total hospitalization and rehabilitation from a medical record review.

\section{Assessment of sleep-disordered breath}

We performed sleep examination in the patient's bed using a portable polysomnography Stardust II (Respironics Inc., Murrysville, PA, USA). This multichannel device recorded five diagnostic parameters: oxygen saturation, pulse rate, nasal airflow, respiratory effort, and body position. We performed the measurement for sleep quality from $9 \mathrm{PM}$ to $6 \mathrm{AM}$, without overnight supervision. A sleep technologist analyzed sleep data, using the American Academy of Sleep Medicine criteria [23]. We excluded patients with a recorded sleep time of less than 180 minutes from the outcome analysis.

We define apnea as an airflow reduction of $\geq 90 \%$ for at least 10 seconds, and hypopnea as an airflow reduction of $\geq 50 \%$ for at least 10 seconds, followed by an oxygen desaturation of $\geq 3 \%$ [23]. We classify apneas with thoracic motion, without thoracic motion, or with an initial lack of motion followed by respiratory effort, as obstructive, central, or mixed, respectively. We define the apnea-hypopnea index (AHI) as the mean number of apneas and hypopneas per hour. We consider an AHI higher than 15 as SDB [24]. We define the obstructive apnea index (OAI) and central apnea index (CAI) as the mean number of obstructive apnea and central apnea events per hour, respectively. We define the oxygen desaturation index (DI) as the mean number of oxygen desaturations of $\geq 3 \%$ per hour. We classify sleep apnea as obstructive or central, according to the type of predominant event. Therefore, predominantly OSA was diagnosed when $>50 \%$ of the respiratory events were of the obstructive type [3].

We evaluated the quality of sleep before stroke onset with the Epworth Sleepiness Scale (ESS) [25]. We obtained answers for the ESS from co-operative patients, or alternatively, from relatives. The ESS assesses daytime sleepiness preceding stroke onset, and it clinically defines a score $>10$ as excessive daytime sleepiness (EDS) [26].

\section{Statistics}

We performed statistical analyses using IBM SPSS Statistics ver. 23.0 (IBM, Armonk, NY, USA). To identify factors associated with SDB in patients, we divided the patients into two groups, according to the presence of SDB. We compared clinical and demographic variables between these groups. We used an independent t-test and the chi-square test to compare continuous data and categorical data between groups, respectively. We tested differences in clinical data between three groups (supratentorial, brainstem, and cerebellum) using a one-way analysis of variance. We used a linear regression analysis to identify independent factors in relation to AHI. We considered a p-value of less than 0.05 as statistically significant.

\section{RESULTS}

Of the 194 stroke patients, we excluded 118 patients and enrolled 76 patients. Consequently, we evaluated 46 patients (61\%) in the outcome analysis, comprising 25 men and 21 women, with a mean age of $65.0 \pm 11.0$ years (Fig. 1). Poor cooperation in the sleep study was the most common cause of withdrawal from the study. The proportion of stroke subtypes was 31 patients with ischemic stroke and 15 patients with hemorrhagic stroke. The mean time

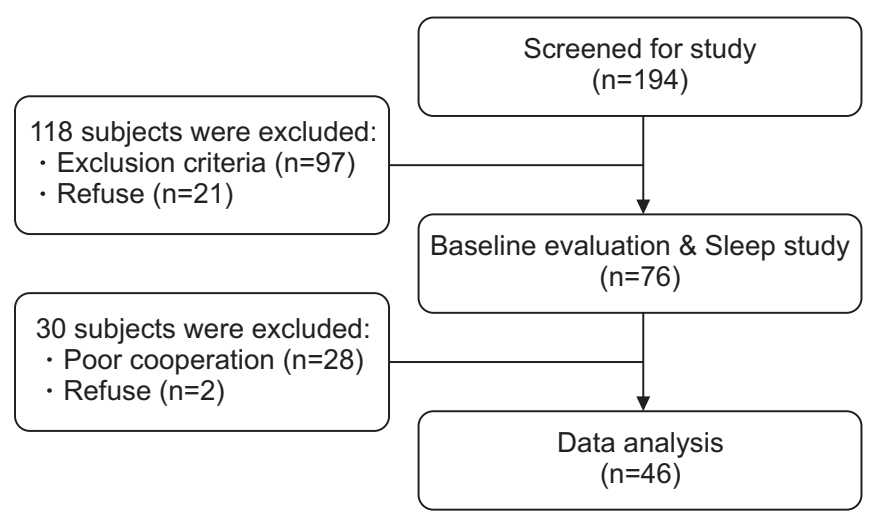

Fig. 1. CONSORT diagram for patient inclusion and exclusion. 
between the sleep study and stroke onset was $35.5 \pm 37.2$ days (range, 7-173 days). The mean values were: AHI, 24.2 \pm 17.0 ; OAI, $9.8 \pm 10.0$; CAI, $4.6 \pm 6.8$; and oxygen DI, $24.4 \pm 18.9$. More specifically, the AHI was $<5$ in $17.4 \%, \geq 5$ in $82.6 \%$, and $\geq 30$ in $32.6 \%$ of the patients, respectively. The overall SDB prevalence (AHI $\geq 15$ ) was $67.4 \%$ (31 of 46). Table 1 summarizes these results.

We found significant differences between patients with and without SDB in the initial FAC, NIHSS, K-MBI, EQ$5 \mathrm{D}$ and changes in mRS between admission and discharge (Table 1). Stroke severity, assessed by the NIHSS, was worse in patients with SDB, compared to without SDB. SDB patients had a lower overall cognitive status (K-MMSE and MoCA-K) than patients without SDB, but this difference was not statistically significant. Baseline mRS did not differ significantly between patients with and without SDB, but $\mathrm{mRS}$ at discharge $(3.5 \pm 1.0$ and
$2.5 \pm 1.1$, respectively; $\mathrm{p}=0.001)$ and changes between admission and discharge $(0.5 \pm 0.6$ and $1.1 \pm 0.7$, respectively; $\mathrm{p}=0.016$ ) showed significant differences between the two groups. There were no significant differences in age, BMI, and systolic or diastolic blood pressure. There was no difference in time between stroke onset and admission to the department of rehabilitation medicine. However, patients with SDB tended to have both a longer hospitalization and rehabilitation than those without SDB. Specifically, stroke patients with SDB spent 5.9 more total days in the hospital than patients without SDB, but this difference was not statistically significant.

Table 2 shows the polysomnographic data. The mean recorded total time was $413.5 \pm 130.2$ minutes. Thirteen patients $(41.9 \%)$ had primarily obstructive apnea. Only five patients (16.1\%) exhibited predominantly central apnea. Thirteen patients (41.9\%) did not show a dominant

Table 1. Baseline characteristics of stroke patients with AHI $\geq 15$ and $<15$

\begin{tabular}{|c|c|c|c|c|}
\hline & All patients $(n=46)$ & $\mathrm{AHI} \geq 15(n=31)$ & AHI $<15(n=15)$ & p-value \\
\hline Age (yr) & $65.0 \pm 11.0$ & $65.3 \pm 11.1$ & $64.4 \pm 11.4$ & 0.796 \\
\hline Gender, male & $25(54.3)$ & $18(58.1)$ & $7(46.7)$ & 0.467 \\
\hline BMI $\left(\mathrm{kg} / \mathrm{m}^{2}\right)$ & $24.5 \pm 3.8$ & $25.1 \pm 3.6$ & $23.4 \pm 4.1$ & 0.196 \\
\hline Ischemic & $31(67.4)$ & $21(67.7)$ & $10(66.7)$ & 0.942 \\
\hline Hypertension & $28(60.9)$ & $22(71.0)$ & $6(40.0)$ & $0.044^{*}$ \\
\hline Diabetes & $14(30.4)$ & $9(29.0)$ & $5(33.3)$ & 0.766 \\
\hline LOS, total & $61.2 \pm 26.3$ & $63.1 \pm 28.5$ & $57.2 \pm 21.2$ & 0.437 \\
\hline LOS, RM & $41.7 \pm 21.3$ & $43.9 \pm 23.8$ & $37.0 \pm 14.5$ & 0.305 \\
\hline FAC & $1.5 \pm 1.0$ & $1.3 \pm 1.1$ & $1.9 \pm 0.9$ & $0.004^{*}$ \\
\hline NIHSS & $6.3 \pm 4.8$ & $7.5 \pm 5.2$ & $3.9 \pm 2.8$ & $0.005^{*}$ \\
\hline K-MMSE & $18.0 \pm 8.9$ & $16.2 \pm 8.9$ & $21.6 \pm 8.2$ & 0.051 \\
\hline K-MBI & $41.7 \pm 24.6$ & $35.8 \pm 24.3$ & $54.1 \pm 21.0$ & $0.013^{*}$ \\
\hline MoCA-K & $13.9 \pm 8.2$ & $12.0 \pm 7.7$ & $17.4 \pm 8.3$ & 0.057 \\
\hline $\mathrm{mRS}$ & $3.9 \pm 0.8$ & $4.0 \pm 0.6$ & $3.5 \pm 0.9$ & 0.234 \\
\hline EQ-5D & $0.192 \pm 0.452$ & $0.091 \pm 0.467$ & $0.400 \pm 0.348$ & $0.017^{*}$ \\
\hline Dysphagia & $21(45.7)$ & $16(51.6)$ & $5(33.3)$ & 0.243 \\
\hline NIHSS at discharge & $4.7 \pm 4.1$ & $5.6 \pm 4.4$ & $2.9 \pm 2.5$ & $0.011^{*}$ \\
\hline $\mathrm{K}-\mathrm{MBI}$ at discharge & $59.9 \pm 24.9$ & $53.4 \pm 26.1$ & $73.3 \pm 15.7$ & $0.009^{*}$ \\
\hline $\mathrm{mRS}$ at discharge & $3.2 \pm 1.1$ & $3.5 \pm 1.0$ & $2.5 \pm 1.1$ & $0.001^{*}$ \\
\hline AHI & $24.2 \pm 17.0$ & $33.1 \pm 13.0$ & $5.6 \pm 4.5$ & $<0.001^{*}$ \\
\hline
\end{tabular}

Values are presented as mean \pm standard deviation or number (\%).

BMI, body mass index; LOS, length of stay; RM, rehabilitation medicine; FAC, Functional Ambulation Category; NIHSS, National Institutes of Health Stroke Scale; K-MMSE, Korean version of Mini-Mental State Examination; K-MBI, Korean version of Modified Barthel Index; MoCA-K, Korean version of Montreal Cognitive Assessment; mRS, Modified Rankin Scale; EQ-5D, EuroQol five dimensions questionnaire; AHI, apnea-hypopnea index. ${ }^{*} \mathrm{p}<0.05$. 
apnea type. Accordingly, the AHI was significantly higher in patients with SDB than without SDB. The OAI, CAI, and DI were also relatively higher in patients with SDB. However, mean heart rate did not differ significantly between the two groups. Premorbid sleep quality, assessed by ESS, showed significantly higher scores in the SDB

Table 2. Polysomnographic data of patients with and without SDB

\begin{tabular}{lccc}
\hline & $\begin{array}{c}\text { Patients } \\
\text { with SDB }\end{array}$ & $\begin{array}{c}\text { Patients } \\
\text { without } \\
\text { SDB }\end{array}$ & p-value \\
\hline AHI & $33.1 \pm 13.0$ & $5.6 \pm 4.5$ & $<0.001^{*}$ \\
OPI & $13.0 \pm 10.7$ & $3.3 \pm 3.4$ & $<0.001^{*}$ \\
CPI & $6.5 \pm 7.5$ & $0.6 \pm 1.2$ & $<0.001^{*}$ \\
HI & $8.7 \pm 7.4$ & $1.6 \pm 2.0$ & $<0.001^{*}$ \\
Mean heart rate & $65.6 \pm 12.7$ & $65.0 \pm 11.5$ & 0.875 \\
DI & $32.9 \pm 17.1$ & $6.8 \pm 5.7$ & $<0.001^{*}$ \\
Mean $\mathrm{O}_{2}$ saturation $(\%)$ & $93.3 \pm 1.9$ & $95.0 \pm 1.4$ & $0.001^{*}$ \\
ESS & $6.1 \pm 4.5$ & $2.3 \pm 2.1$ & $<0.001^{*}$ \\
\hline
\end{tabular}

Values are presented as mean \pm standard deviation.

SDB, sleep-disordered breathing; AHI, apnea-hypopnea index; OAI, obstructive apnea index; CAI, central apnea index; HI, hypopnea index; DI, desaturation index; ESS, Epworth Sleepiness Scale.

${ }^{*} \mathrm{p}<0.05$. group.

There were no significant differences in AHI, OAI or CAI by stroke type (Table 3 ). Hemorrhagic stroke patients tended to show a longer period of total hospitalization than ischemic stroke patients. Brainstem stroke patients showed the lowest CAI, but this difference was not statistically significant.

In the regression analysis concerning factors showing an independent association with AHI, we found the initial NIHSS ( $\beta=0.358$; 95\% confidence interval $[\mathrm{CI}]$, $0.318-2.214 ; \mathrm{p}=0.010)$ and premorbid ESS $(\beta=0.368 ; 95 \%$ $\mathrm{CI}, 0.399-2.553 ; \mathrm{p}=0.008$ ) to be significantly and independently correlated with AHI. There was no significant association between AHI and age, gender, type and location of the stroke, BMI, or blood pressure (Table 4).

Table 4. Independent predictor of AHI

\begin{tabular}{lccc}
\hline & $\boldsymbol{\beta}$ & adjusted $\mathbf{R}^{2}$ & $\mathbf{p}$-value \\
\hline AHI & & 0.258 & \\
NIHSS (admission) & 0.358 & & $0.010^{*}$ \\
ESS (premorbid) & 0.368 & & $0.008^{*}$ \\
\hline
\end{tabular}

AHI, apnea-hypopnea index; NIHSS, National Institutes of Health Stroke Scale; ESS, Epworth Sleepiness Scale. ${ }^{*} \mathrm{p}<0.05$.

Table 3. Sleep indices and clinical characteristic in etiologic stroke types

\begin{tabular}{|c|c|c|c|c|c|c|c|}
\hline & \multicolumn{3}{|c|}{ Stroke type } & \multicolumn{4}{|c|}{ Location of stroke lesion } \\
\hline & $\begin{array}{c}\text { Ischemic } \\
(n=31)\end{array}$ & $\begin{array}{c}\text { Hemorrhagic } \\
(n=15)\end{array}$ & p-value & $\begin{array}{c}\text { Supratentorial } \\
(n=31)\end{array}$ & $\begin{array}{c}\text { Brainstem } \\
(n=11)\end{array}$ & $\begin{array}{c}\text { Cerebellum } \\
(n=4)\end{array}$ & p-value \\
\hline Age (yr) & $66.2 \pm 10.4$ & $62.5 \pm 12.3$ & 0.326 & $62.9 \pm 11.4$ & $72.1 \pm 5.2$ & $62.0 \pm 14.2$ & $0.047^{*}$ \\
\hline LOS, total & $55.9 \pm 27.6$ & $71.9 \pm 20.0$ & $0.032^{*}$ & $65.2 \pm 26.8$ & $46.2 \pm 21.7$ & $71.2 \pm 21.8$ & 0.084 \\
\hline LOS, RM & $38.1 \pm 21.4$ & $49.1 \pm 19.7$ & 0.093 & $42.4 \pm 21.6$ & $34.6 \pm 20.0$ & $55.7 \pm 17.7$ & 0.221 \\
\hline $\operatorname{BMI}\left(\mathrm{kg} / \mathrm{m}^{2}\right)$ & $24.9 \pm 3.6$ & $23.8 \pm 4.3$ & 0.421 & $24.5 \pm 4.1$ & $24.6 \pm 2.6$ & $24.5 \pm 4.9$ & 0.996 \\
\hline NIHSS & $5.8 \pm 5.0$ & $7.3 \pm 4.4$ & 0.299 & $7.0 \pm 5.4$ & $5.8 \pm 2.6$ & $2.0 \pm 1.4$ & 0.135 \\
\hline K-MMSE & $19.2 \pm 9.0$ & $15.5 \pm 8.5$ & 0.195 & $15.3 \pm 9.4$ & $23.0 \pm 4.8$ & $25.3 \pm 3.0$ & $0.009^{*}$ \\
\hline K-MBI & $45.0 \pm 26.4$ & $35.0 \pm 19.6$ & 0.158 & $40.0 \pm 24.6$ & $39.6 \pm 25.6$ & $61.8 \pm 16.9$ & 0.239 \\
\hline AHI & $23.3 \pm 17.1$ & $25.9 \pm 17.3$ & 0.639 & $24.8 \pm 18.1$ & $23.3 \pm 16.8$ & $21.4 \pm 9.8$ & 0.915 \\
\hline OAI & $8.5 \pm 7.0$ & $12.5 \pm 14.4$ & 0.207 & $9.6 \pm 11.3$ & $9.8 \pm 6.1$ & $11.8 \pm 10.0$ & 0.922 \\
\hline CAI & $5.0 \pm 7.3$ & $3.7 \pm 5.6$ & 0.498 & $5.1 \pm 7.7$ & $2.7 \pm 2.9$ & $5.6 \pm 6.7$ & 0.570 \\
\hline DI & $23.2 \pm 18.7$ & $26.9 \pm 19.9$ & 0.546 & $25.7 \pm 20.3$ & $22.7 \pm 17.7$ & $18.7 \pm 11.8$ & 0.747 \\
\hline
\end{tabular}

Values are presented as mean \pm standard deviation or number (\%).

LOS, length of stay; RM, rehabilitation medicine; BMI, body mass index; NIHSS, National Institutes of Health Stroke Scale; K-MMSE, Korean version of Mini-Mental State Examination; K-MBI, Korean version of Modified Barthel Index; AHI, apnea-hypopnea index; OAI, obstructive apnea index; CAI, central apnea index; DI, desaturation index. ${ }^{*} \mathrm{p}<0.05$. 


\section{DISCUSSION}

This study seeks to estimate the prevalence of SDB in Korean patients with subacute stroke. Parra et al. [20] reported that the prevalence of SDB was $71 \%$ in the acute stage and $62 \%$ in the subacute stage. Similarly, we found a high prevalence $(67.4 \%)$ of SDB in our patients who were admitted to a rehabilitation unit, consistent with those of previous western studies. We performed our sleep study in the subacute phase of stroke using a portable polysomnography. Most previous studies have dealt with acute stroke patients. However, most of the patients admitted to rehabilitation medicine have moderate to severe neurologic deficits and require a relatively longer period of hospitalization. Our study has the advantage of allowing the prediction of long-term SDB changes, as well as functional changes in moderate-to-severe stroke patients.

We found that patients with SDB had a significantly worse neurological status, cognitive function, and functional independence, compared to patients without SDB. This was the case not only at admission to rehabilitation, but also at discharge from the hospital. Thus, our study showed the short-term influence of SDB on the functional recovery. Baseline mRS did not differ significantly between patients with and without SDB, but $\mathrm{mRS}$ at discharge and changes between admission and discharge showed significant differences between the two groups. Patients with SDB had the worse functional capacity, as measured by the mRS, compared to patients without SDB. This finding concurs with those of previous studies $[10,12,27]$, which showed that SDB was associated with worse functional outcome at discharge, and at 3,6, and 12 months after stroke. Although there was no statistically significant difference, patients with SDB had a longer period of hospitalization than patients without SDB, in agreement with a previous study [12]. The relatively slow recovery of stroke patients with SDB might be contributed to prolonged hospitalization.

We failed to find any significant difference in prevalence and clinical characteristics of SDB according to stroke type or location. Brown et al. [28] found that the presence of an acute infarction in the brainstem is associated with both the existence and severity of SDB. However, a previous study performed in Korea found no difference in AHI across locations, including the brainstem [1]. This discrepancy in findings is most probably due to difficulties in measuring AHI in patients with severe brainstem stroke and differences in patient characteristics between studies. In our study, hemorrhagic stroke patients tended to show a longer period of total hospitalization than ischemic stroke patients, which could be attributed to a comparatively longer period of acute care required for hemorrhagic stroke patients [29]. While hemorrhagic stroke patients were hospitalized for 5 days more than ischemic stroke patients before transfer to the department of rehabilitation medicine, the length of stay in the rehabilitation unit did not show a significant difference.

Sleep studies were not performed before the stroke; it was not possible to determine whether sleep apnea or stroke came first. Previous studies suggested that sleep apnea more likely precedes the onset of stroke and that there is an improvement in SDB in most patients after the acute phase of stroke $[10,20]$. In this study, premorbid sleep quality, assessed by ESS, showed significantly high scores in the SDB group. Furthermore, premorbid ESS was significantly related to AHI by regression analysis. We found that ESS could adequately predict a high likelihood of SDB in the stroke patients. Also, the severity of EDS preceding stroke (as estimated by the ESS) was a predictor of stroke severity. These findings may indicate that SDB precedes the stroke event in most cases. Our study does not allow identifying the nature and cause of SDB. Still, it can provide important data on the subacute phase of stroke.

A few limitations of our study should be noted. First, the use of a portable polysomnography tends to underestimate AHI. The portable device, without electroencephalography recordings, cannot differentiate between waking and sleeping states; thus, it may underestimate respiratory events. Consequently, the prevalence of SDB may be higher than our results indicate. Second, the small sample size and the characteristics of the patients referred to our hospital should be considered when interpreting our findings. The patients admitted to the department of rehabilitation medicine had moderate-tosevere neurologic deficits and a higher NIHSS score on admission. Moreover, we excluded recurrent stroke. In addition, more than half of the eligible patients did not participate because of poor cooperation. Thus, due to the strict inclusion and exclusion criteria, our results may not be representative of all stroke patients. Lastly, patients 
with higher neurologic deficits showed more severe SDB. The severity of stroke may have a direct impact on the results. Future studies should focus on identifying the exact causes of SDB in stroke patients and elucidate the effect of initial stroke severity on the sleep study.

In conclusion, SDB is common in subacute stroke patients admitted for rehabilitation and is associated with worse neurologic and functional status. This study emphasized that SDB can affect the neurologic and functional status of stroke patients. Clinicians must assess the sleep status of stroke patients and attempt to appropriately treat the sleep disorder. Also, a portable polysomnography can be performed in the rehabilitation process of stroke patients and provide important data for successful rehabilitation.

\section{CONFLICT OF INTEREST}

No potential conflict of interest relevant to this article was reported.

\section{ACKNOWLEDGMENTS}

This study was supported by grants (HI10C2020, HI13C1990) from the Korean Health Technology R\&D Project, Ministry of Health \& Welfare, from NRF2015R1C1A1A01055923, NRF-2017R1A2B4006500 and VitalAire Korea Inc.

\section{REFERENCES}

1. Ahn SH, Kim JH, Kim DU, Choo IS, Lee HJ, Kim HW. Interaction between sleep-disordered breathing and acute ischemic stroke. J Clin Neurol 2013;9:9-13.

2. Klobucnikova K, Siarnik P, Carnicka Z, Kollar B, Turcani P. Causes of excessive daytime sleepiness in patients with acute stroke: a polysomnographic study. J Stroke Cerebrovasc Dis 2016;25:83-6.

3. Aaronson JA, van Bennekom CA, Hofman WF, van Bezeij T, van den Aardweg JG, Groet E, et al. Obstructive sleep apnea is related to impaired cognitive and functional status after stroke. Sleep 2015;38:1431-7.

4. Mohsenin V, Valor R. Sleep apnea in patients with hemispheric stroke. Arch Phys Med Rehabil 1995;76: 71-6.

5. Martinez-Garcia MA, Galiano-Blancart R, Soler-Cata- luna JJ, Cabero-Salt L, Roman-Sanchez P. Improvement in nocturnal disordered breathing after first-ever ischemic stroke: role of dysphagia. Chest 2006;129: 238-45.

6. Yan-fang S, Yu-ping W. Sleep-disordered breathing: impact on functional outcome of ischemic stroke patients. Sleep Med 2009;10:717-9.

7. Heinzer R, Vat S, Marques-Vidal P, Marti-Soler H, Andries D, Tobback N, et al. Prevalence of sleep-disordered breathing in the general population: the HypnoLaus study. Lancet Respir Med 2015;3:310-8.

8. Kim J, In K, Kim J, You S, Kang K, Shim J, et al. Prevalence of sleep-disordered breathing in middle-aged Korean men and women. Am J Respir Crit Care Med 2004;170:1108-13.

9. Harbison J, Ford GA, James OF, Gibson GJ. Sleep-disordered breathing following acute stroke. QJM. 2002; 95:741-7.

10. Bassetti CL, Milanova M, Gugger M. Sleep-disordered breathing and acute ischemic stroke: diagnosis, risk factors, treatment, evolution, and long-term clinical outcome. Stroke 2006;37:967-72.

11. Camilo MR, Schnitman SV, Sander HH, Eckeli AL, Fernandes RM, Leite JP, et al. Sleep-disordered breathing among acute ischemic stroke patients in Brazil. Sleep Med 2016;19:8-12.

12. Kaneko Y, Hajek VE, Zivanovic V, Raboud J, Bradley TD. Relationship of sleep apnea to functional capacity and length of hospitalization following stroke. Sleep 2003;26:293-7.

13. Balfors EM, Franklin KA. Impairment of cerebral perfusion during obstructive sleep apneas. Am J Respir Crit Care Med 1994;150(6 Pt 1):1587-91.

14. Netzer N, Werner P, Jochums I, Lehmann M, Strohl KP. Blood flow of the middle cerebral artery with sleep-disordered breathing: correlation with obstructive hypopneas. Stroke 1998;29:87-93.

15. Bradley TD, Floras JS. Obstructive sleep apnoea and its cardiovascular consequences. Lancet 2009;373:8293.

16. Arzt M, Young T, Finn L, Skatrud JB, Bradley TD. Association of sleep-disordered breathing and the occurrence of stroke. Am J Respir Crit Care Med 2005;172: 1447-51.

17. Bassetti C, Aldrich MS. Sleep apnea in acute cerebrovascular diseases: final report on 128 patients. Sleep 
1999;22:217-23.

18. Shahar E, Whitney CW, Redline S, Lee ET, Newman $A B$, Nieto FJ, et al. Sleep-disordered breathing and cardiovascular disease: cross-sectional results of the Sleep Heart Health Study. Am J Respir Crit Care Med 2001;163:19-25.

19. Bassetti C, Aldrich MS, Chervin RD, Quint D. Sleep apnea in patients with transient ischemic attack and stroke: a prospective study of 59 patients. Neurology 1996;47:1167-73.

20. Parra O, Arboix A, Bechich S, Garcia-Eroles L, Montserrat JM, Lopez JA, et al. Time course of sleep-related breathing disorders in first-ever stroke or transient ischemic attack. Am J Respir Crit Care Med 2000;161(2 Pt 1):375-80.

21. Epstein LJ, Kristo D, Strollo PJ Jr, Friedman N, Malhotra A, Patil SP, et al. Clinical guideline for the evaluation, management and long-term care of obstructive sleep apnea in adults. J Clin Sleep Med 2009;5:263-76.

22. Kepplinger J, Barlinn K, Albright KC, Schrempf W, Boehme AK, Pallesen LP, et al. Early sleep apnea screening on a stroke unit is feasible in patients with acute cerebral ischemia. J Neurol 2013;260:1343-50.

23. Berry RB, Budhiraja R, Gottlieb DJ, Gozal D, Iber C, Kapur VK, et al. Rules for scoring respiratory events in sleep: update of the 2007 AASM Manual for the Scoring of Sleep and Associated Events. Deliberations of the Sleep Apnea Definitions Task Force of the American Academy of Sleep Medicine. J Clin Sleep Med 2012;8:597-619.

24. Collop NA, Anderson WM, Boehlecke B, Claman D, Goldberg R, Gottlieb DJ, et al. Clinical guidelines for the use of unattended portable monitors in the diagnosis of obstructive sleep apnea in adult patients: Portable Monitoring Task Force of the American Academy of Sleep Medicine. J Clin Sleep Med 2007;3:737-47.

25. Cho YW, Lee JH, Son HK, Lee SH, Shin C, Johns MW. The reliability and validity of the Korean version of the Epworth sleepiness scale. Sleep Breath 2011;15:37784.

26. Johns MW. Sleepiness in different situations measured by the Epworth Sleepiness Scale. Sleep 1994;17:70310.

27. Good DC, Henkle JQ, Gelber D, Welsh J, Verhulst S. Sleep-disordered breathing and poor functional outcome after stroke. Stroke 1996;27:252-9.

28. Brown DL, McDermott M, Mowla A, De Lott L, Morgenstern LB, Kerber KA, et al. Brainstem infarction and sleep-disordered breathing in the BASIC sleep apnea study. Sleep Med 2014;15:887-91.

29. Kim SM, Hwang SW, Oh EH, Kang JK. Determinants of the length of stay in stroke patients. Osong Public Health Res Perspect 2013;4:329-41. 